\title{
Symmetry and states of self stress in triangulated toroidal frames
}

\author{
P.W. Fowler \\ School of Chemistry, University of Exeter \\ Stocker Road, Exeter EX4 4QD, UK \\ S.D. Guest \\ Department of Engineering, University of Cambridge \\ Trumpington Street, Cambridge, CB2 1PZ, UK \\ January 15, 2002

\begin{abstract}
A symmetry extension of Maxwell's rule for rigidity of frames shows that every fully triangulated torus has at least six states of self stress with a well defined set of symmetries related to rotational and translational representations in the point group. In contrast, in trivalent polyhedral toroidal frames, i.e. the duals of the deltahedra, the rigid body motions plus the mechanisms are at least equal in number to the bars, and their symmetry representation contains that of a set of vector slides along the bars.
\end{abstract}

\section{Introduction}

Maxwell's rule expresses a condition for the determinacy of a pin-jointed frame (Maxwell, 1864). If an unsupported, three-dimensional frame composed of rigid bars connected via frictionless joints is statically and kinematically determinate, the number of bars $b$ must be at least $3 j-6$, where $j$ is the number of joints. In general, the frame will support $s$ states of self-stress (bar tensions in the absence of load) and $m$ mechanisms (joint displacements without bar extension), where

$$
s-m=b-3 j+6
$$


(Calladine, 1978). Recently, this algebraic formula has been shown (Fowler and Guest, 2000) to be the scalar aspect of a symmetry version of Maxwell's rule,

$$
\Gamma(s)-\Gamma(m)=\Gamma(b)-\Gamma(j) \otimes \Gamma_{T}+\Gamma_{T}+\Gamma_{R}
$$

where $\Gamma(q)$ is the reducible representation of the set $\{q\}$ of bars, joints, mechanisms etc. and $\Gamma_{T}, \Gamma_{R}$ are the translational and rotational representations, all within the point group of the frame. All quantities in (2) can be computed by standard manipulations of the character table of the group (Atkins, Child and Phillips, 1970). Equation (2) is, in effect, a set of Maxwell conditions, each concerning an aspect of the symmetry defined by one of the irreducible representations of the point group, thus giving additional insight into the allowed stresses and motions of the frame.

Maxwell (1870) pointed out a number of consequences of (1) for certain classes of frameworks. In particular he showed that the number of states of self-stress must equal the number of mechanisms for a spherical deltahedron, and that a toroidal deltahedron must have at least six states of self-stress. In this paper, we extend these results by pointing out the consequences of (2) for the triangulated toroids, and their duals. It is proved that six states of self stress, of defined symmetry, are present in every fully triangulated toroidal frame. For their duals, the trivalent polyhedral frames of toroidal topology, mechanisms are characterised by number and symmetry using similar grouptheoretical reasoning.

\section{Spherical polyhedral frames}

The key to the results in this paper is the symmetry counterpart of Euler's theorem for polyhedra. Although the symmetry extension of Maxwell's rule given in (2) is valid for all frames, extra information can be gleaned when the frame has a specific embedding; this Section will consider the example when all vertices lie on a surface deformable to that of a sphere (i.e. implying spherical topology but not a specific geometry). Section 3 will then examine the case of toroidal frames.

The scalar Euler theorem for a spherical polyhedron connects the numbers of vertices, faces and edges by

$$
v+f=e+2 .
$$

The combination $v-e+f$ is known as the Euler characteristic. For an orientable surface, $v-e+f=2-2 g$, where $g$ is the genus (Stillwell, 1993). In the case of the sphere, $g=0$ and $v-e+f=2$. 
The symmetry extension of the Euler equation for $g=0$ (Ceulemans and Fowler, 1991) connects reducible representations associated with the three types of structural component

$$
\Gamma(v)+\Gamma(f) \otimes \Gamma_{\epsilon}=\Gamma_{\|}(e)+\Gamma_{0}+\Gamma_{\epsilon}
$$

where $\Gamma(v)$ and $\Gamma(f)$ are permutation representations of the sets of vertices and face centres and $\Gamma_{\|}(e)$ is the reducible representation of a set of vectors running along the edges. The one-dimensional representations $\Gamma_{0}$ and $\Gamma_{\epsilon}$ are respectively the totally symmetric and the pseudoscalar representations in the point group of the frame; $\Gamma_{0}$ has character +1 under all operations of the point group, whereas $\Gamma_{\epsilon}$ has character +1 under all proper and -1 under all improper operations.

Fully triangulated spherical polyhedra (i.e. spherical deltahedra) have $e=3 v-6$, and the symmetry equivalent of this second counting relation is (Ceulemans and Fowler, 1991)

$$
\Gamma(e)=\Gamma(v) \otimes \Gamma_{T}-\Gamma_{T}-\Gamma_{R}
$$

which, when applied to a polyhedral frame of bars $(b \equiv e)$ and joints $(j \equiv$ $v$ ), shows that the states of self-stress and the mechanisms of a spherical deltahedral frame are equisymmetric, i.e.

$$
\Gamma(s)=\Gamma(m) .
$$

The well known generic rigidity of the deltahedron (Graver, Servatius and Servatius, 1993) implies that $s$ and $m$ must be equal in number; (6) further implies a detailed match, symmetry by symmetry.

A dual relation for trivalent polyhedral frames, i.e. those in which every joint connects three bars, is (Ceulemans and Fowler, 1991)

$$
\Gamma(v) \otimes \Gamma_{T}=\Gamma(e)+\Gamma_{\|}(e)
$$

which implies that, in a spherical, trivalent, polyhedral frame, the representations of the states of self-stress and the mechanisms differ by

$$
\Gamma(s)-\Gamma(m)=\Gamma_{T}+\Gamma_{R}-\Gamma_{\|}(e)
$$

Equation (8) shows that such a frame will in general have at least $3 j / 2-6$ mechanisms spanning the symmetries of inextensional 'slides' along the bars minus those of the six rigid-body motions. This division of internal coordinates into slides and stretches has been proposed as the basis for a treatment of molecular vibrations (Ceulemans et al., 2001). In the analogy between frames and molecules, bar extensions correspond to stretches, mechanisms to angle bends, twists and torsions, and states of self-stress to redundancies in the internal-coordinate force field. 


\section{Toroidal polyhedral frames}

In its most symmetrical form, the toroidal surface has point group $D_{\infty h}$, denoting the presence of a principal axis of infinite order, an infinite number of reflection planes containing that axis, a reflection plane normal to it and containing an infinite number of binary axes, and the inversion and identity elements. Other automorphisms of the torus, for example the 'anapole' rotation that exchanges inner and outer equatorial circles, do not correspond to point-group operations. A toroidal frame, therefore, belongs to a finite subgroup of $D_{\infty h}$ i.e. one of $D_{n h}, D_{n d}, D_{n}, C_{n v}, C_{n h}, C_{n}$, or the trivial groups $C_{s}, C_{i}, C_{1}$.

On the torus, the scalar Euler theorem is

$$
v+f=e
$$

where the Euler characteristic $v-e+f$ is now zero, as the genus of the torus is 1 . The symmetry extension of (9) is more complicated than before in that the Euler characteristic of zero is now replaced by a self-cancelling grouping of four one-dimensional representations:

$$
\Gamma(v)+\Gamma(f) \otimes \Gamma_{\epsilon}+\Gamma_{z}+\Gamma_{z} \otimes \Gamma_{\epsilon}=\Gamma_{\|}(e)+\Gamma_{0}+\Gamma_{\epsilon}
$$

(Ceulemans and Fowler, 1995). In (10), $\Gamma_{z}$ is the representation of a translation along the central axis of the torus. In the point group of the underlying toroidal surface, $D_{\infty h}$, the four terms $\Gamma_{0}, \Gamma_{z}, \Gamma_{z} \otimes \Gamma_{\epsilon}, \Gamma_{\epsilon}$ constitute the full list of one-dimensional representations $\Sigma_{g}^{+}, \Sigma_{u}^{+}, \Sigma_{g}^{-}, \Sigma_{u}^{-}$(Atkins et al. (1970)). 1995)

The equivalent of (5) for a toroidal deltahedron is (Ceulemans and Fowler,

$$
\Gamma(e)=\Gamma(v) \otimes \Gamma_{T}-\Gamma_{T}-\Gamma_{R}+\Gamma_{T} \otimes \Gamma_{z}+\Gamma_{R} \otimes \Gamma_{z}
$$

which reduces to the scalar counting rule $e=3 v$ applicable to triangulated surfaces with an Euler characteristic of zero. Comparison with Maxwell's rule, (2), yields the result that for a fully triangulated toroidal frame, the representations of states of self-stress and mechanisms differ by

$$
\Gamma(s)-\Gamma(m)=\left(\Gamma_{T}+\Gamma_{R}\right) \otimes \Gamma_{z}
$$

In the parent $D_{\infty h}$ group, the RHS of (12) evaluates to $\Sigma_{g}^{+}+\Sigma_{g}^{-}+\Pi_{g}+\Pi_{u}$, which is the direct sum of $\Gamma_{0}, \Gamma_{\epsilon}$ and the symmetries of the translations along the two axes orthogonal to the main axis of the torus, $\Gamma\left(T_{x}, T_{y}\right)$, and rotations about these axes, $\Gamma\left(R_{x}, R_{y}\right)$.

In a general subgroup of $D_{\infty h}, \Gamma(s)-\Gamma(m)$ for a toroidal deltahedron has characters $\chi(E)=6, \chi(C(\phi))=2(1+2 \cos \phi)$ for rotation $\phi$ around the 
principal axis, $\chi\left(C_{2}^{\prime}\right)=2$ for rotation about a primary axis, and $\chi(R)=0$ for improper operations $R$. In the subgroups with a principal axis of order 3 or higher, each pair of states descended from a $\Pi$ representation in $D_{\infty h}$ remains degenerate. In the pure rotational sub groups $C_{n}$ and $D_{n}$ there is no distinction in symmetry between $\Gamma_{0}$ and $\Gamma_{\epsilon}$, and $\Gamma(s)-\Gamma(m)=2 \Gamma_{T} \otimes \Gamma_{z}$.

Thus, a toroidal deltahedron has been proved to have at least six states of self-stress of these fixed symmetries, as was promised in the Introduction. The distribution of stresses in the six states of self-stress are shown in Figure 1. The operation of taking the dual, which interchanges vertices with face centres and rotates all edges by $\pi / 2$ in the embedding surface, is also defined for the torus. An example of a trivalent frame on a torus would be a fully hexagonal covering of the surface. It turns out that equation (7) also applies unchanged to the vertices and edges of the trivalent polyhedron that results from dualisation of the toroidal deltahedron. Hence, in a toroidal, trivalent, polyhedral frame, the representations of the state of self-stress and the mechanisms differ by

$$
\Gamma(s)-\Gamma(m)=\Gamma_{T}+\Gamma_{R}-\Gamma_{\|}(e)
$$

Again, an analysis of the $3 j / 2-6$ mechanisms in terms of 'slides' along the bars is possible.

\section{Example}

As a particular example of the results regarding toroidal frames, consider the frame illustrated in Figure 2. In the $D_{3 h}$ group appropriate to this frame, $\Gamma_{0} \equiv A_{1}^{\prime}, \Gamma\left(T_{x}, T_{y}\right) \equiv E^{\prime}, \Gamma_{\epsilon} \equiv A_{1}^{\prime \prime}, \Gamma\left(R_{x}, R_{y}\right) \equiv E^{\prime \prime}$ (Atkins et al. (1970)), and hence $\Gamma(s)-\Gamma(m)=A_{1}^{\prime}+E^{\prime}+A_{1}^{\prime \prime}+E^{\prime \prime}$. This is shown below for the particular framework in Figure 2 using the explicit calculation of the LHS of (12) using the general Maxwell relation (2) (see (Fowler and Guest, 2000) for similar schemes).

\begin{tabular}{r|rrrrrr}
$D_{3 h}$ & $E$ & $2 C_{3}$ & $3 C_{2}^{\prime}$ & $\sigma_{h}$ & $2 S_{3}$ & $3 \sigma_{v}$ \\
\hline$-\Gamma(j)$ & -12 & 0 & -2 & -6 & 0 & -4 \\
$\otimes \Gamma_{T}$ & +3 & 0 & -1 & +1 & -2 & +1 \\
\hline$-\Gamma(j) \otimes \Gamma_{T}$ & -36 & 0 & +2 & -6 & 0 & -4 \\
$+\Gamma_{T}+\Gamma_{R}$ & +6 & 0 & -2 & 0 & 0 & 0 \\
\hline$-\Gamma(j) \otimes \Gamma_{T}+\Gamma_{T}+\Gamma_{R}$ & -30 & 0 & 0 & -6 & 0 & -4 \\
$+\Gamma(b)$ & +36 & 0 & +2 & +6 & 0 & +4 \\
\hline$=\Gamma(s)-\Gamma(m)$ & +6 & 0 & +2 & 0 & 0 & 0
\end{tabular}


This reduces, as expected, to

$$
\begin{aligned}
A_{1}^{\prime}+E^{\prime}+A_{1}^{\prime \prime}+E^{\prime \prime} & \equiv\left(\Gamma_{T}+\Gamma_{R}\right) \otimes \Gamma_{z} \\
& \equiv \Gamma_{z} \otimes \Gamma_{z}+\Gamma\left(T_{x}, T_{y}\right) \otimes \Gamma_{z}+\Gamma\left(R_{z}\right) \otimes \Gamma_{z}+\Gamma\left(R_{x}, R_{y}\right) \otimes \Gamma_{z} \\
& \equiv \Gamma_{0}+\Gamma\left(R_{x}, R_{y}\right)+\Gamma_{\epsilon}+\Gamma\left(T_{x}, T_{y}\right)
\end{aligned}
$$

Detailed prediction of the expected states of self stress can be carried out by using the decomposition of the structure into orbits. An orbit is a set of symmetry-equivalent points that interconvert under the operations of the group. Each finite group $G$ has only a limited set of possible orbits, and each orbit is characterised by a site group $H$, which is the subgroup of operations generated by the symmetry elements that pass though one member of the orbit. It is easily seen that the total size of the orbit is the ratio of the orders of the two groups: $|G| /|H|$.

In the $D_{3 h}$ group, the possible orbits of points are (Fowler and Quinn, 1986): $O_{1}$ (a single point at the group centre, $H=D_{3 h}$ ); $O_{2}$ (two points on the $C_{3}$ axis, $H=C_{3 v}$ ); $O_{3}$ (three points in the $\sigma_{h}$ plane, $\left.H=C_{2 v}\right) ; O_{6 h}(\operatorname{six}$ points on the $\sigma_{h}$ plane, $H=C_{s}$ ); $O_{6 v}$ (six points forming a trigonal prism, $H=C_{s}$ ); $O_{12}$ (twelve points forming a $D_{3 h}$ hexagonal prism, $H=C_{1}$ ).

The 36 bars of the present structure fall into 5 orbits. There are two $O_{3}$ orbits of bars (in Figure 2(b), bars $\{7-8,8-9,9-7\}$ and bars $\{4-5$ 5-6 6-4 $\}$ ), each making a contribution to $\Gamma(b)$ according to

$$
\Gamma\left(b, O_{3}\right)=A_{1}^{\prime}+E^{\prime},
$$

There is one $O_{6 v}$ orbit (bars $\{1-2,2-3,3-1,10-11,11-12,12-10\}$ ) making a contribution to $\Gamma(b)$ according to

$$
\Gamma\left(b, O_{6 v}\right)=A_{1}^{\prime}+E^{\prime}+A_{2}^{\prime \prime}+E^{\prime \prime},
$$

There are two $O_{12}$ orbits (bars $\{1-7,2-7,2-8,3-8,3-9,1-9,7-10,7-11,8-11$, 8-12, 9-12, 9-10 $\}$ and bars $\{1-4,2-4,2-5,3-5,3-6,1-6,4-10,4-11,5-11,5-12$, $6-12,6-10\})$ each making a contribution to $\Gamma(b)$ according to

$$
\Gamma\left(b, O_{12}\right)=A_{1}^{\prime}+A_{2}^{\prime}+2 E^{\prime}+A_{1}^{\prime \prime}+A_{2}^{\prime \prime}+2 E^{\prime \prime},
$$

Taking the scalar product of $\Gamma(b)\left(=2 \Gamma\left(b, O_{3}\right)+\Gamma\left(b, O_{6 v}\right)+2 \Gamma\left(b, O_{12}\right)\right)$ with $\Gamma(s)-\Gamma(m)$ shows that the 6 necessary states of self-stress are defined by a total of 19 parameters ( 5 for $A_{1}^{\prime}, 7$ for $E^{\prime}, 2$ for $A_{1}^{\prime \prime}, 5$ for $E^{\prime \prime}$ ). The symmetry-adapted linear combinations of these parameters describing the bar forces for each orbit are easily found by inspection, and are shown in Figure 3. The general patterns of signs for the uniform torus from Figure 1 
are thus converted into specific patterns of signs and magnitudes shown in Figure 3 by taking into account the orbit structure of the frame.

The final step, determination of the actual values of the 19 parameters for the 6 states of self-stress, depends on the particular geometry of the framework. This analysis could be carried out using full symmetry-adapted bases, as described by Kangwai and Guest (2000), or with the methods based on orbits described by Bossavit (1993), but was in fact performed by finding the states of self-stress in a cartesian coordinate system (Pellegrino, 1993), and then projecting these onto the symmetry-adapted set. This calculation confirms that, for this geometry, the states of self-stress given by the symmetry calculation are the only states of self-stress. The patterns of tension and compression in the bars follow Figure 3, with the parameter values reported in Table 2.

In keeping with the necessary-but-not-sufficient nature of Maxwell's rule, extra states of self-stress may be induced in particular other geometries of a similar framework, as when a vertex is flattened into coplanarity with all of its neighbours, but these are then compensated by equisymmetric mechanisms and make no change to the balance $\Gamma(s)-\Gamma(m)$.

\section{Conclusion}

The symmetry extension of Maxwell's rule can give specific information on states of self-stress and mechanisms for frames with non-trivial topologies. The case of toroidal frames has been explored here.

It is worth noting that the results generalise to frames embedded in surfaces of any genus. Thus, any orientable surface topologically equivalent to a sphere with $g$ handles will have at least $6 g$ states of self-stress when the frame is fully triangulated. Their symmetries will again be invariant functions of $g$. In a double torus or pretzel-like frame, for example, the 12 enforced states of self stress $\operatorname{span}\left(\Gamma_{T}+\Gamma_{R}\right) \otimes\left(\Gamma_{T}-\Gamma_{z}\right)$ (Ceulemans and Fowler, 1995). The dual trivalent polyhedral frame always obeys equation (13), irrespective of genus.

A general formulation for any $g$ is available (Ceulemans, Szopa and Fowler, 1996). Its application is limited only by the ingenuity of engineers in designing frames of sufficiently complex topology. 


\section{References}

Atkins, P.W., Child, M.S. and Phillips, C.S.G., 1970. Tables for Group Theory. OUP, Oxford.

Bossavit, A., 1993. On the computation of strains and stresses in symmetrical articulated structures. Lectures in Applied Mathematics, American Mathematical Society 29, 111-123.

Calladine, C.R., 1978. Buckminster Fuller's 'Tensegrity' structures and Clerk Maxwell's rules for the construction of stiff frames. Int. J. Solids Structures $14,161-172$.

Ceulemans, A. and Fowler, P.W., 1991. Extension of Euler's theorem to the symmetry properties of polyhedra. Nature 353, 52-54.

Ceulemans, A. and Fowler, P.W., 1995. Symmetry extensions of Euler's theorem for polyhedral, toroidal and benzenoid molecules, J. Chem. Soc. Faraday Trans. 91, 3089-93.

Ceulemans, A., Titeca, B.C., Chibotaru, L.F., Vos, I. and Fowler, P.W., 2001. Complete force fields for trivalent and deltahedral cages: group theory and applications to cubane, closo-dodecahedrane and buckminsterfullerene. Journal of Physical Chemistry A 105, 8284-8295.

Ceulemans, A., Szopa, M. and Fowler, P.W., 1996. Homology groups, symmetry representations and polyhedral clusters. Europhys. Lett. 36, 645-649.

Fowler, P.W. and Guest, S.D., 2000. A symmetry extension of Maxwell's rule for rigidity of frames. Int. J. Solids Structures 37, 1793-1804.

Fowler, P.W. and Quinn, C.M., 1986. $\sigma, \pi$ and $\delta$ representations of the molecular point groups. Theoretica Chimica Acta 70, 333-350.

Graver, J.E, Servatius, B., and Servatius, H., 1993. Combinatorial Rigidity. Graduate Studies in Mathematics, AMS, Providence.

Kangwai, R.D. and Guest, S.D., 2000. Symmetry-adapted equilibrium matrices. Int. J. Solids Structures 37, 1525-1548

Maxwell, J.C., 1864. On the calculation of the equilibrium and stiffness of frames, Phil. Mag. 27, 294-299. Also: Collected papers, XXVI. Cambridge University Press, 1890.

Maxwell, J.C., 1870. On reciprocal figures, frames, and diagrams of forces, Trans. Royal Soc. Edinburgh 26, 1-40. Also: Collected papers, XXXIX. Cambridge University Press, 1890. 
Pellegrino, S., 1993. Structural computations with the singular value decomposition of the equilibrium matrix. Int. J. Solids Structures 30, 3025-3035.

Stillwell, J., 1993. Classical topology and combinatorial group theory, 2nd. edition. Graduate Texts in Mathematics, Springer, New York. 


\section{Figures}

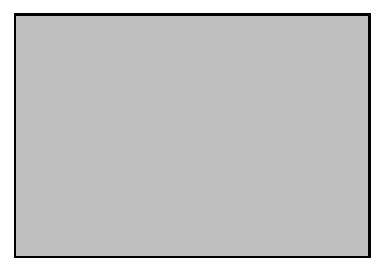

(a)

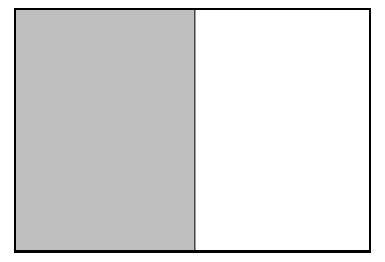

(b)

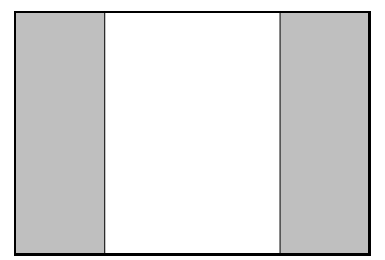

(c)

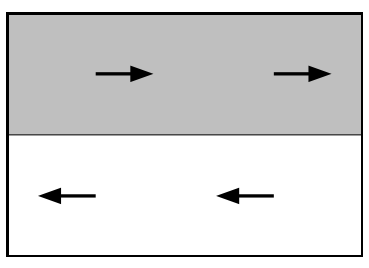

(d)

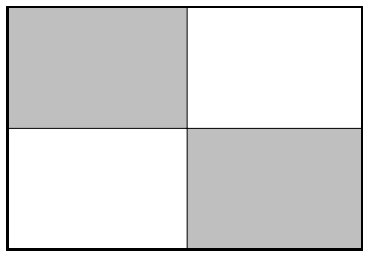

(e)

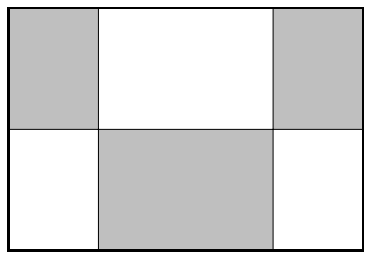

(f)

Figure 1. The six necessary states of self-stress of the toroidal deltahedron. The standard rectangular representation is used (Stillwell, 1993), where the top and bottom edges are to be identified, as are left and right edges. The top and bottom edges correspond to the inner equator of the torus. The simplest function on the torus showing the planes forced by symmetry are shown; in each case shaded regions are of opposite sign to unshaded: (a) $\Gamma_{0}$; (b), (c) $\Gamma\left(T_{x}, T_{y}\right)$; (d) $\Gamma_{\epsilon}$, counter-rotations about the principal axis in opposed senses above and below the equatorial nodal plane; (e),(f) $\Gamma\left(R_{x}, R_{y}\right)$. 


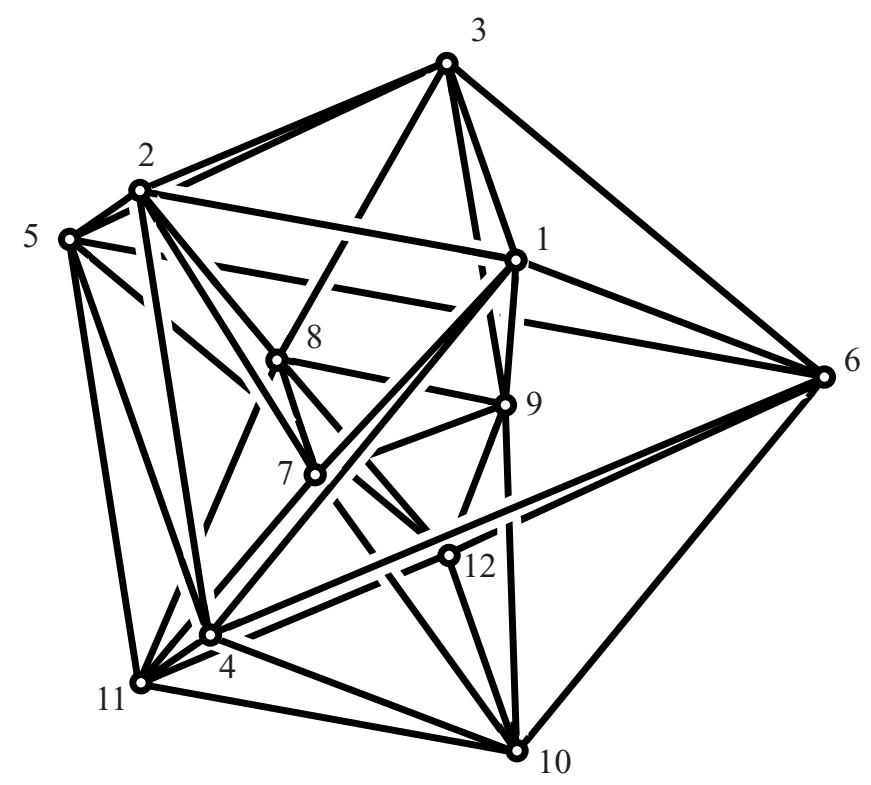

(a)

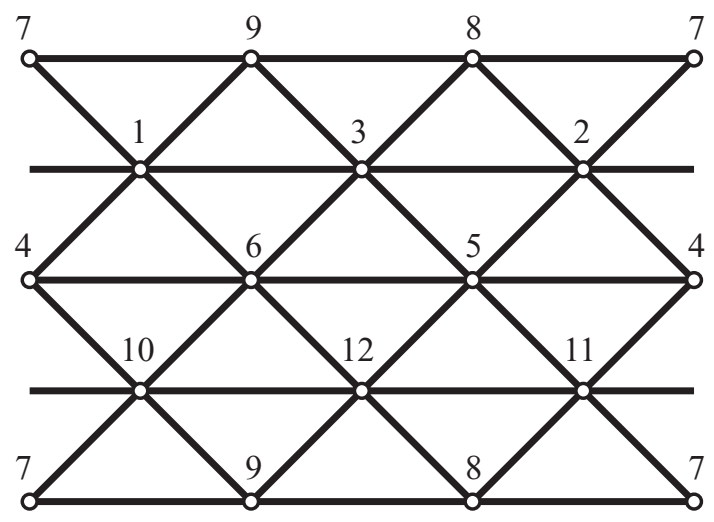

(b)

Figure 2. Example toroidal frame with maximal $D_{3 h}$ symmetry, having 12 joints and 36 bars: (a) The bars and joints; (b) The rectangular representation. A numbering scheme is shown for the joints: the cartesian coordinates of the joints are given in Table 1. The triangles 1-2-3 7-8-9 10-11-12 are not faces of the toroidal polyhedron, but they are boundary circuits of the central tunnel of the torus 


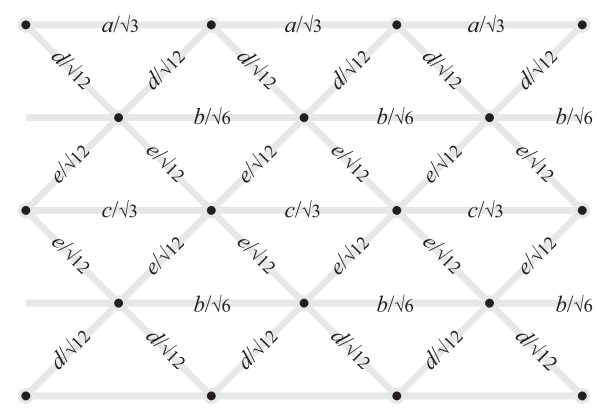

(a)

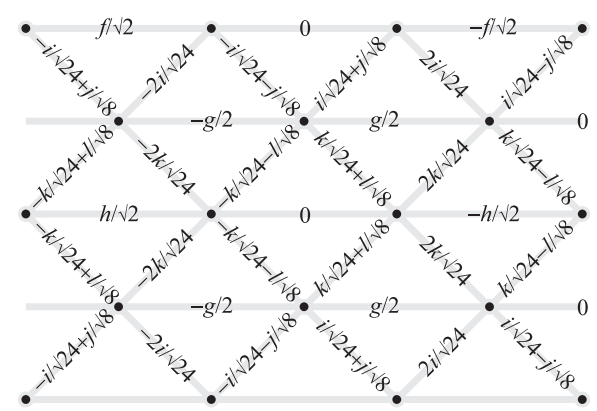

(b)

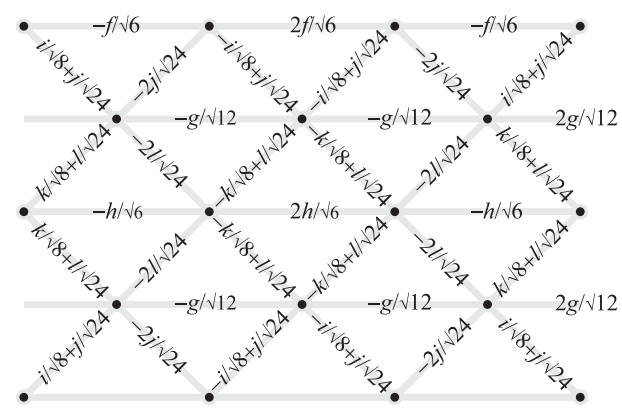

(c)

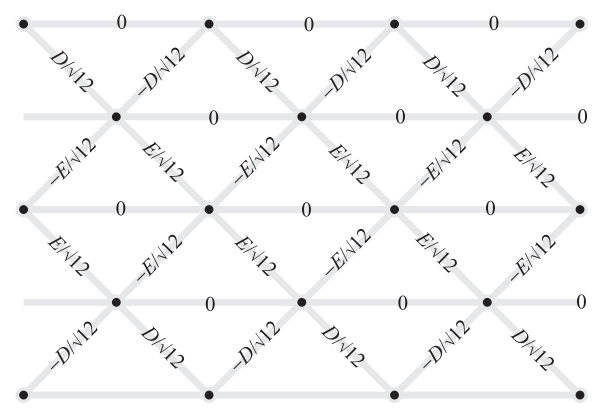

(d)

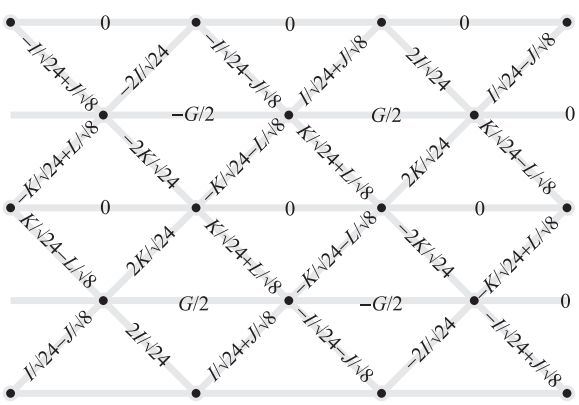

(e)

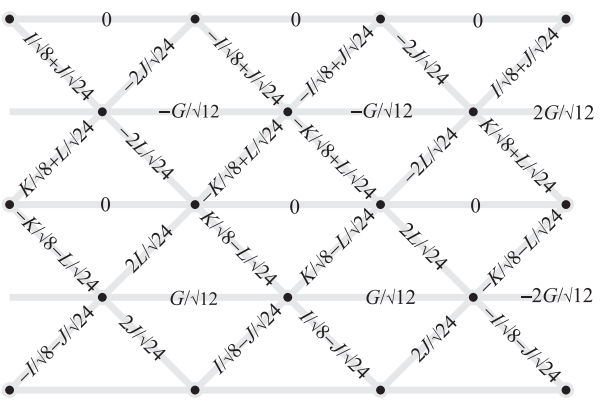

(f)

Figure 3. The independent states of self-stress for the example frame. (a)(f) correspond to the general patterns of Figure 1, and show the symmetryallowed patterns of signs and magnitudes defined on the five orbits of bars present in this frame. Lower-case letters are used for the parameters of states that are even under reflection in the horizontal mirror plane $\left(A_{1}^{\prime}\right.$ and $\left.E^{\prime}\right)$, and upper-case is used for the odd $A_{1}^{\prime \prime}$ and $E^{\prime \prime}$ states. Note that both components of a degenerate state are specified by a single set of parameters, since degenerate components mix under the operations of the group. Calculated values of the 19 parameters for the particular geometry of Table 1 are listed in Table 2. 


\section{Tables}

\begin{tabular}{|c|rrr|}
\hline joint & \multicolumn{1}{|c}{$x$} & \multicolumn{1}{c|}{$y$} & \multicolumn{1}{c|}{$z$} \\
\hline 1 & -0.500 & 0.289 & 0.707 \\
2 & 0.500 & 0.289 & 0.707 \\
3 & 0 & -0.577 & 0.707 \\
4 & 0 & 1.386 & 0 \\
5 & 1.200 & -0.693 & 0 \\
6 & -1.200 & -0.693 & 0 \\
7 & 0 & 0.346 & 0 \\
8 & 0.300 & -0.173 & 0 \\
9 & -0.300 & -0.173 & 0 \\
10 & -0.500 & 0.289 & -0.707 \\
11 & 0.500 & 0.289 & -0.707 \\
12 & 0 & -0.577 & -0.707 \\
\hline
\end{tabular}

Table 1. The coordinates of the joints of the toroidal deltahedron shown in Figure 2.

\begin{tabular}{|c|c|r||c|c|c|}
\hline Symmetry & Parameter & Value & Symmetry & Parameter & Value \\
\hline & $a$ & 0.031 & & & \\
$\Gamma_{0}=A_{1}^{\prime}$ & $b$ & 0.200 & & & \\
& $c$ & -0.597 & $\Gamma_{\epsilon}=A_{1}^{\prime \prime}$ & & \\
& $d$ & -0.409 & & $D$ & -0.928 \\
& $e$ & 0.659 & & $E$ & -0.373 \\
\hline & $f$ & -0.091 & & & \\
& $g$ & 0.347 & & $G$ & -0.408 \\
& $h$ & 0.432 & & & \\
& $i$ & -0.547 & $\Gamma\left(R_{x}, R_{y}\right)=E^{\prime \prime}$ & $I$ & 0.241 \\
& $j$ & -0.237 & & $J$ & -0.417 \\
& $k$ & 0.468 & & $K$ & -0.388 \\
& $l$ & -0.334 & & $L$ & 0.672 \\
\hline
\end{tabular}

Table 2. Parameters for the 6 states of self stress in the toroidal deltahedron with the joint coordinates given in Table 1 . The parameters are defined in Figure 3. Within each state of self-stress, all parameters can be multiplied by the same arbitrary constant. 